\title{
Resonant Tunneling and Coulomb Oscillations
}

\author{
Jürgen König ${ }^{1}$, Herbert Schoeller ${ }^{1,2}$, and Gerd Schön ${ }^{1}$ \\ ${ }^{1}$ Institut für Theoretische Festkörperphysik, Universität Karlsruhe, 76128 Karlsruhe, Germany \\ ${ }^{2}$ Department of Physics, Simon Fraser University, Burnaby, B.C., V5A 1S6, Canada
}

(June 1, 1995)

\begin{abstract}
The influence of quantum fluctuations on electron transport through small metallic islands with Coulomb blockade effects is studied beyond the perturbative regime. In tunnel junctions with low resistance higher order coherent processes and "inelastic resonant tunneling" become important. We present a path integral real-time description, which allows a systematic diagrammatic classification of these processes. Quantum fluctuations renormalize system parameters and lead to finite lifetime broadening. Both effects are observable in the nonlinear conductance. The finite bias voltage introduces an energy scale up to which quantum fluctuations are probed. It can be larger than the temperature, which makes quantum effects experimentally observable.

73.40.Gk., 73.40Rw., 71.10.+x
\end{abstract}

Typeset using REVTEX 
Electron transport through mesoscopic metallic islands is strongly influenced by the charging energy associated with the low capacitance of the junctions [1-3]. A variety of single-electron phenomena, including Coulomb blockade and oscillations of the conductance as a function of a gate voltage have been observed. A master equation description of sequential tunneling is sufficient as long as the conductance of the barriers is low, $\alpha_{0} \equiv h /\left(4 \pi^{2} e^{2} R_{T}\right) \ll 1$. In general, quantum fluctuations and higher order coherent tunneling processes should be considered [4-9]. This includes "cotunneling" [10], where in a second order coherent process electrons tunnel via a virtual state of the island, as well as resonant tunneling. In comparison to the case of independent electrons we encounter here two complications: (i) In metallic systems with overwhelming probability different electron states are involved in the different transitions of the coherent process (this is denoted by "inelastic"). (ii) The strong Coulomb interaction cannot be accounted for perturbatively.

In this article we develop a systematic diagrammatic technique to identify the processes of sequential tunneling, cotunneling and inelastic resonant tunneling. We study the time evolution of the density matrix. In an earlier paper [9] we had formulated the problem, after a separation of charge and fermionic degrees of freedom, in a many-body expansion technique. Here we reformulate it in a real-time path-integral representation [11]. The latter is familiar from the studies of dissipation in quantum mechanics [12,13]. Dissipation associated with tunneling of electrons was investigated in Refs. [14,3]. Similar problems arise in the context of local, strongly correlated Fermi systems like the Kondo and Anderson model.

As examples we study the single electron transistor and the electron box. For a detailed description see for instance [2]. In the transistor a metallic island is coupled by two tunnel junctions $(L, R)$ to two electrodes, and further capacitively to a gate voltage $V_{G}$. A transport voltage $V=V_{L}-V_{R}$ drives a current. The charging energy $E_{c h}(n)=\left(n e-Q_{G}\right)^{2} /(2 C)$ depends on the number of excess electrons $n$ on the island and on the continuously varying external charge $Q_{G}=C_{G} V_{G}+C_{L} V_{L}+C_{R} V_{R}$. The total island capacitance $C=C_{L}+C_{R}+C_{G}$ defines the energy scale $E_{C} \equiv e^{2} / 2 C$. The electron box, consisting of an island coupled via a 
tunnel junction to one electrode and capacitively to the gate voltage, is described similarly.

At low temperature the electron number $n\left(Q_{G}\right)$ in the box increases in unit steps by tunneling as $Q_{G}$ is increased. In the transistor tunneling occurs in lowest order perturbation theory only if the electrochemical potential of one electrode is high enough to allow an electron to enter the island, while the electrochemical potential of the other electrode allows the tunneling process to that electrode, i.e. $e V_{L}>E_{c h}(n+1)-E_{c h}(n)>e V_{R}$. Within this window the current is $4 R_{t} I\left(V, Q_{G}\right)=V-4\left[Q_{G}-(n+1 / 2) e\right]^{2} /\left(C^{2} V\right)$. I.e. the nonlinear conductance, $G\left(V, Q_{G}\right)=\partial I / \partial V$, shows as a function of $Q_{G}$ an e-periodic series of structures of width $C V$ with, at $T=0$, vertical steps at its edges. At finite temperature $\left\langle n\left(Q_{G}\right)\right\rangle$ and the steps are washed out.

Quantum fluctuations further wash out these steps. Below we describe these processes diagrammatically. In the most interesting case we can re-sum the diagrams and obtain closed expressions for the stationary density matrix and the spectral density describing the charge excitations of the system. Our main findings are: (i) Quantum fluctuations renormalize the energy and conductance. (ii) The coherent resonant tunneling processes give rise to a broadening of the charge state levels, which is observable in the nonlinear conductance.

The description of the single electron transistor is based on the Hamiltonian $H=H_{L}+$ $H_{R}+H_{I}+H_{c h}+H_{t, L}+H_{t, L}$. The first terms describe noninteracting electrons in the metallic left and right lead and island. These are treated as reservoirs, i.e. their distributions are assumed to be thermal with electrochemical potential which depends on the state. The Coulomb interaction $H_{c h}$ depends on the charge on the island, as expressed by $E_{c h}(n)$. Charge transfer is described by tunneling Hamiltonians $H_{t, r}=\sum_{k \in r, q \in I, \sigma} T_{r} c_{k \sigma}^{\dagger} c_{q \sigma}+$ h.c. , where $r=L, R$. The matrix elements and densities of states determine the tunneling conductances, $R_{r}^{-1}=\left(4 \pi e^{2} / \hbar\right) N_{r}(0) N_{I}(0)\left|T_{r}\right|^{2}$. We consider "wide" metallic junctions with many transverse channels $N_{c h} \gg 1$. Hence "inelastic" higher order tunneling processes, involving different electron states for each step, dominate over "elastic" processes which involve the same state repeatedly.

We describe the system of interacting electrons in a path integral representation. A 
Hubbard-Stratonovich transformation, used to handle the capacitive interaction, introduces as collective variable the phase $\varphi$, the quantum mechanical conjugates of the charge on the island. The phases of the electrodes are fixed by the applied voltages, $\varphi_{r}=e V_{r} t$. Next, the electronic degrees of freedom can be traced out, followed by an expansion of the electron propagators. Since we consider wide junctions, only simple loops need to be retained. Their iteration introduces in each order a factor $N_{c h}$, hence they dominate over more complicated higher order loops. After this stage the system is described by a reduced density matrix $\rho\left(\left\{\varphi_{1}\right\},\left\{\varphi_{2}\right\}\right)$. It can be expressed by an effective action, which depends on the phases $\varphi_{\sigma}$ corresponding to the forward and backward propagator $\sigma=1,2$. The structure of the theory is familiar from Refs. [13,12], where a quantum system coupled to a harmonic oscillator bath has been considered, and from Refs. [14,3], where electron tunneling has been described. The tunneling couples the forward and backward propagators. For each junction we have [14,3]

$$
S_{t, r}\left[\varphi_{1}, \varphi_{2}\right]=4 \pi i \sum_{\sigma, \sigma^{\prime}=1,2} \int_{t_{i}}^{t_{f}} d t \int_{t_{i}}^{t} d t^{\prime} \alpha_{r}^{\sigma, \sigma^{\prime}}\left(t-t^{\prime}\right) \cos \left[\varphi_{\sigma}(t)-\varphi_{\sigma^{\prime}}\left(t^{\prime}\right)\right]
$$

The kernels are given in Fourier space by $\alpha_{r}^{\sigma, 1}(\omega)=(-1)^{\sigma+1} \alpha_{r}^{-}(\omega), \alpha_{r}^{\sigma, 2}(\omega)=(-1)^{\sigma} \alpha_{r}^{+}(\omega)$ with $\alpha_{r}^{ \pm}(\omega)= \pm \alpha_{0, r}\left(\omega-e V_{r}\right)\left[\exp \left( \pm \beta\left(\omega-e V_{r}\right)\right)-1\right]^{-1}$.

An important step for a systematic description of tunneling processes is the change from the phase to a charge representation [3,11], accomplished by $(\hbar=k=1)$

$$
\begin{aligned}
& \rho\left(t_{f} ; n_{1 f}, n_{2 f}\right)= \\
& \sum_{n_{1 i}, n_{2 i}} \rho\left(t_{i} ; n_{1 i}, n_{2 i}\right) \int d \varphi_{1 f} d \varphi_{2 f} d \varphi_{1 i} d \varphi_{2 i} \int_{\varphi_{1 i}}^{\varphi_{1 f}} \mathcal{D} \varphi_{1}(t) \int_{\varphi_{2 i}}^{\varphi_{2 f}} \mathcal{D} \varphi_{2}(t) \int \mathcal{D} n_{1}(t) \int \mathcal{D} n_{2}(t) \\
& \exp \left\{i \sum_{r} S_{t, r}\left[\varphi_{1}, \varphi_{2}\right]+\sum_{\sigma=1,2}(-1)^{\sigma}\left(i n_{\sigma i} \varphi_{\sigma i}-i n_{\sigma f} \varphi_{\sigma f}+i S_{c h}\left[n_{\sigma}\right]-i \int d t n_{\sigma} \dot{\varphi}_{\sigma}\right)\right\} .
\end{aligned}
$$

The charging energy is $S_{c h}\left[n_{\sigma}\right]=\int_{t_{i}}^{t_{f}} d t\left(n_{\sigma} e-Q_{G}\right)^{2} /(2 C)$. In systems with discrete charges the integrations include a summation over winding numbers [3]. Next we expand the tunneling terms $\exp \left(i S_{t}\right)$ and integrate over $\varphi_{\sigma}$. Each of the exponentials $\exp \left[ \pm i \varphi_{\sigma}(t)\right]$ describes tunneling of an electron at time $t$ on the forward or backward branch, $\sigma=1$ or 2, respectively. These changes occur in pairs in each junction, $r=L, R$, and are connected by $\alpha_{r}^{\sigma, \sigma^{\prime}}\left(t-t^{\prime}\right)$. 
Each term of the expansion can be visualized by a diagram. Several examples are displayed in Fig. (1). There is a closed time-path consisting of two horizontal lines, corresponding to the forward and backward propagator between $t_{i}$ to $t_{f}$. Along the time-path vertices are arranged, connected in pairs by tunneling lines.

We start from a density matrix which is diagonal, $\rho\left(t_{i}\right)=P^{(0)}(n)|n\rangle\langle n|$. The sum of all diagrams, starting and ending in the diagonal states $n$ and $n^{\prime}$, respectively, is denoted by $\Pi_{n, n^{\prime}}$. It can be expressed by the free propagator $\Pi^{(0)}$ and an irreducible self-energy $\Sigma_{n, n^{\prime}}$, which describes the transitions,

$$
\Pi_{n, n^{\prime}}=\Pi_{n}^{(0)}+\sum_{n^{\prime \prime}} \Pi_{n, n^{\prime \prime}} \Sigma_{n^{\prime \prime}, n^{\prime}} \Pi_{n^{\prime}}^{(0)}
$$

We identify the solution of Eq.(3), multiplied with $P^{(0)}(n)$, as the stationary distribution function $\sum_{n} P^{(0)}(n) \Pi_{n, n^{\prime}}=P^{s t}\left(n^{\prime}\right)$. The sum rule $\sum_{n^{\prime}} \Sigma_{n, n^{\prime}}=0$ (see Ref. [11]) implies

$$
0=\sum_{n^{\prime}}\left[-P^{s t}(n) \Sigma_{n, n^{\prime}}+P^{s t}\left(n^{\prime}\right) \Sigma_{n^{\prime}, n}\right]
$$

I.e., we recover a stationary master equation and note that the stationary solution $P^{s t}(n)$ is independent of the initial distribution $P^{(0)}(n)$. In general the irreducible self-energy $\Sigma_{n^{\prime}, n}$ yields the transition rates of all possible correlated tunneling processes. We reproduce the well-known single electron tunneling (cotunneling) rates by evaluating all diagrams which contain no overlapping tunneling lines (two tunneling lines overlapping in time), as shown in Fig. (1).

The perturbative approach is sufficient for $\alpha_{0} \ln \left(E_{C} / \max \{e V / 2,2 \pi T\}\right) \ll 1$. At larger values of the conductance resonant tunneling processes get important. To proceed we have to find a systematic criterion which diagrams to retain. For this we note that during a tunneling process the reservoirs contain an electron excitation. Our criterion is to take into account only matrix elements of the density matrix which differ at most by two excitations in the leads or (equivalently) in the island. This means that in the diagrams any vertical line cuts at most two tunneling lines. We, furthermore, concentrate on situations where only two charge states, $n=0,1$, need to be considered. This is sufficient when the energy 
difference of the two states $\Delta_{0} \equiv E_{c h}(1)-E_{c h}(0)$ and the bias voltage $e V=e V_{L}-e V_{R}$ are low compared to $E_{C}$. The two restrictions imply that the diagrams contain no crossing tunneling lines, which allows us to evaluate the irreducible self-energy analytically. Using the notations $\alpha_{r}(\omega)=\alpha_{r}^{+}(\omega)+\alpha_{r}^{-}(\omega), \alpha(\omega)=\sum_{r} \alpha_{r}(\omega)$ and $\alpha_{0}=\sum_{r} \alpha_{0, r}$ we find

$$
\Sigma_{0,1}=-\Sigma_{0,0}=2 \pi i \frac{\lambda_{+}}{\lambda} \quad, \quad \Sigma_{1,0}=-\Sigma_{1,1}=2 \pi i \frac{\lambda_{-}}{\lambda}
$$

with $\quad \lambda_{ \pm}=\int d \omega \alpha^{ \pm}(\omega)|\pi(\omega)|^{2} \quad, \quad \lambda=\int d \omega|\pi(\omega)|^{2}$,

and $\quad \pi(\omega)=\frac{1}{\omega-\Delta_{0}-\sigma(\omega)} \quad, \quad \sigma(\omega)=-\int d \omega^{\prime} \frac{\alpha\left(\omega^{\prime}\right)}{\omega^{\prime}-\omega-i \eta}$.

Inserting these quantities in Eq.(4) we arrive at the stationary, normalized probabilities $P_{0}^{s t}=\lambda_{-}$and $P_{1}^{s t}=\lambda_{+}$, with $\lambda_{+}+\lambda_{-}=1$.

The expression for the current at time $t$ in the junction $r$ can be written as

$$
I_{r}(t)=4 \pi i e \int_{-\infty}^{t} d t^{\prime} \sum_{\sigma} \alpha_{r}^{1, \sigma}\left(t-t^{\prime}\right)\left\langle\sin \left[\varphi_{1}(t)-\varphi_{\sigma}\left(t^{\prime}\right)\right]\right\rangle
$$

where the expectation value is taken with the density matrix discussed above, with $t=t_{f}$. We, therefore, study the correlation functions describing charge transfer at times $t$ and $t^{\prime}$

$$
C^{>}\left(t, t^{\prime}\right)=-i\left\langle e^{-i \varphi(t)} e^{i \varphi\left(t^{\prime}\right)}\right\rangle \quad, \quad C^{<}\left(t, t^{\prime}\right)=i\left\langle e^{i \varphi\left(t^{\prime}\right)} e^{-i \varphi(t)}\right\rangle
$$

We further introduce a spectral density for charge excitations $A(\omega)=\left[C^{<}(\omega)-C^{>}(\omega)\right] / 2 \pi i$. Within the approximations described above we find

$$
\begin{aligned}
C^{\zeta}(\omega) & = \pm 2 \pi i \sum_{r} \alpha_{r}(\omega) f\left[ \pm\left(\omega-e V_{r}\right)\right]|\pi(\omega)|^{2}, \\
A(\omega) & =\alpha(\omega)|\pi(\omega)|^{2} \\
\text { and } \quad I_{r} & =\frac{e}{h} 4 \pi^{2} \int d \omega \sum_{r^{\prime}} \frac{\alpha_{r^{\prime}}(\omega) \alpha_{r}(\omega)}{\alpha(\omega)} A(\omega)\left[f\left(\omega-e V_{r^{\prime}}\right)-f\left(\omega-e V_{r}\right)\right] .
\end{aligned}
$$

These results satisfy conservation laws and sum rules, $\sum_{r} I_{r}=0$ and $\int d \omega A(\omega)=1$, and the equilibrium relations between correlation functions and the spectral density. The classical result is recovered in lowest order in $\alpha_{0}$, where $A^{(0)}(\omega)=\delta\left(\omega-\Delta_{0}\right)$. Quantum fluctuations yield energy renormalization and broadening effects, which enter in the spectral density via the complex self-energy $\sigma(\omega)$ given in Eq.(7). 
In equilibrium, for $V_{R}=V_{L}$, the single electron transistor is equivalent to the box. The average electron number becomes $\langle n\rangle=\int d \omega f(\omega) A(\omega)$. In the classical limit it reduces to $\left\langle n^{c l}\right\rangle=f\left(\Delta_{0}\right)$, where the energy difference $\Delta_{0}=E_{C}\left(1-2 C_{G} V_{G} / e\right)$ depends on the gate voltage. It shows a step at $Q_{G}=e / 2$, which is smeared by temperature. At larger values of $\alpha_{0}$ or lower temperature we have to include the self-energy $\sigma(\omega)$ in the spectral density. The limits $T, V=0$ and $|\omega| \leq T, V$ can be analyzed analytically. In the former,

$$
\begin{aligned}
& A(\omega) \cong \frac{|\omega|}{\Delta_{0}} \cdot \frac{\tilde{\Delta}(\omega) \tilde{\alpha}(\omega)}{[\omega-\tilde{\Delta}(\omega)]^{2}+[\pi \tilde{\Delta}(\omega) \tilde{\alpha}(\omega)]^{2}}, \\
& \tilde{\Delta}(\omega)=\frac{\Delta_{0}}{1+2 \alpha_{0} \ln \left(\frac{E_{C}}{|\omega|}\right)} \cdot \frac{1}{1+\pi^{2} \tilde{\alpha}(\omega)^{2}}, \\
& \tilde{\alpha}(\omega)=\frac{\alpha_{0}}{1+2 \alpha_{0} \ln \left(\frac{E_{C}}{|\omega|}\right)} .
\end{aligned}
$$

The spectral density $A(\omega)$ has a maximum at the renormalized energy difference $\Delta$, obtained from the self-consistent solution of $\Delta=\tilde{\Delta}(\Delta)[8,5]$. It is broadened by $\pi \Delta \alpha$, where $\alpha=\tilde{\alpha}(\Delta)$. Due to quantum fluctuations the step of the average charge $\langle n\rangle$ in the electron box at the degeneracy point $\Delta_{0}=0$ is washed out even for $T=0$. At finite $T$ the slope at $\Delta_{0}=0$ shows an anomalous temperature dependence, $\partial\langle n\rangle /\left.\partial \Delta_{0}\right|_{\Delta_{0}=0} \cong$ $-\left\{4 T\left[1+2 \alpha_{0} \ln \left(E_{C} /(2 \pi T)\right)\right]^{2}\right\}^{-1}$.

A pronounced and experimentally accessible signature of quantum fluctuations is contained in the differential conductance $G\left(V, Q_{G}\right)=\partial I(V) / \partial V$ of a transistor at finite voltages. Here we find a renormalization of $\Delta$ and $\alpha_{0}$ by a factor $Z$ with $Z^{-1}=$ $1+2 \alpha_{0} \ln \left(E_{C} / \max \{e V / 2,2 \pi T\}\right)$ and a broadening given by $Z \alpha(\omega)$. Now $e V$ provides an energy scale, and renormalization and life-time effects are probed over a wide energy range even at zero temperature. The result of Eq.(12) is plotted in Fig.(2). For comparison we also show the classical result. Fig.(2) clearly displays the renormalization effects and, moreover, the finite life-time broadening. The width of the structure as a function of $\Delta_{0} / V$ is $Z^{-1}$, which depends logarithmically on $V$. These effects should be observable in an experiment with realistic parameters. Since a transport voltage can be applied which exceeds the temperature, $k T \ll e V \leq E_{C}$, thermal smearing is weak, and quantum effects are visible. 
In conclusion, we have presented a systematic description of coherent single electron tunneling processes including resonant tunneling in a single electron transistor. The nonlinear conductance displays renormalization effects and lifetime broadening induced by these quantum fluctuations.

We acknowledge discussions with G. Falci, A.D. Zaikin and G. Zimanyi and support by the 'Sonderforschungsbereich 195' of the DFG and the Swiss Natl. Science Foundation. 
FIGURES

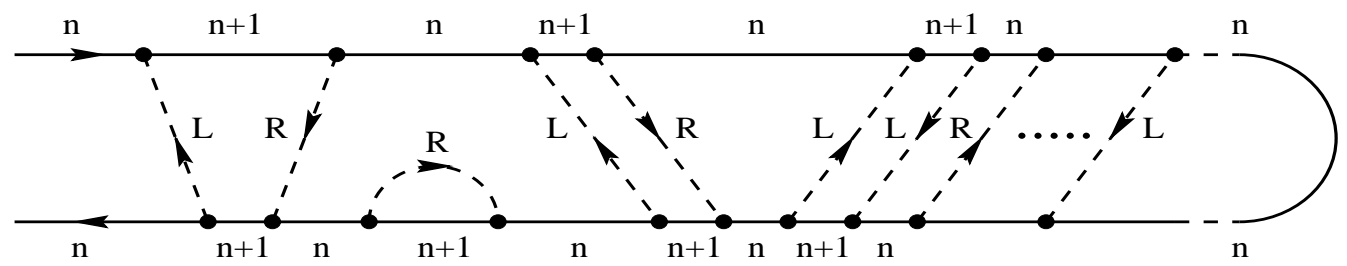

FIG. 1. A diagram showing various tunneling processes: sequential tunneling in the left and right junctions, a term preserving the norm, a cotunneling process, and resonant tunneling.

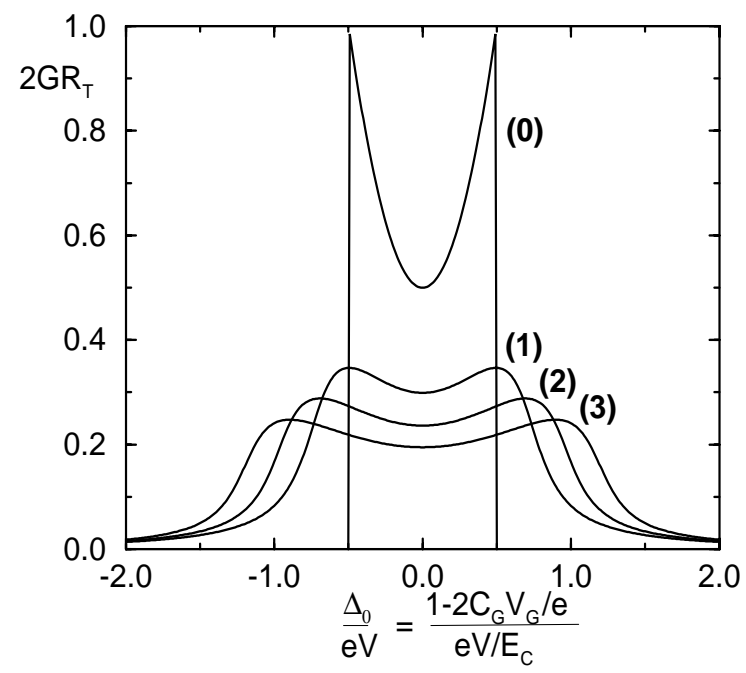

FIG. 2. The differential conductance at $T=0$ in the nonlinear response regime as function of the gap energy normalized to the transport voltage $V$. We choose $\alpha_{0, L}=\alpha_{0, R}=0.05$, and (1) to $(3) e V / E_{C}=0.1,0.01,0.001$. For comparison, (0) shows the result of perturbation theory. 


\section{REFERENCES}

[1] D.V. Averin and K.K. Likharev, in Mesoscopic Phenomena in Solids, ed. B.L. Altshuler et al. (Elsevier, 1991), p. 173

[2] Single Charge Tunneling, NATO ASI Series 294, H. Grabert and M.H. Devoret, eds., (Plenum Press, 1992);

[3] G. Schön and A.D. Zaikin, Phys. Rep. 198, 237 (1990)

[4] P. Lafarge et al., Z. Phys. B - Condensed Matter 85, 327 (1991)

[5] L.I. Glazman and K.A. Matveev, Sov. Phys. JETP 71, 1031 (1990)

[6] D.S. Golubev and A.D. Zaikin, Phys. Rev B 50, 8736 (1994)

[7] H. Grabert, Phys. Rev. B 50, 17364 (1994)

[8] G. Falci, G. Schön and G.T. Zimanyi, Phys. Rev. Lett. 74, 3257 (1995)

[9] H. Schoeller and G. Schön, Phys. Rev. B 50, 18436 (1994)

[10] D.V. Averin and Yu.V. Nazarov, Chapter 6 in Ref. [2]

[11] J. König, H. Schoeller, G. Schön and R. Fazio, in "Quantum Dynamics of Submicron Structures", edited by H. A. Cerdeira, B. Kramer and G. Schön, NATO ASI Series E, Vol. 291 (Kluwer, Dordrecht, 1995), p.221

[12] A.O. Caldeira and A.J. Leggett, Ann. Phys. (N.Y.) 149, 374 (1983)

[13] R.P. Feynman and F.L. Vernon, Ann. Phys. (N.Y.) 24, 118 (1963)

[14] U. Eckern, G. Schön and V. Ambegaokar, Phys. Rev. B 30, 6419 (1984) 